\section{Trisenox: a paradigm shift in APL therapy, an interview with Francesco Lo-Coco}

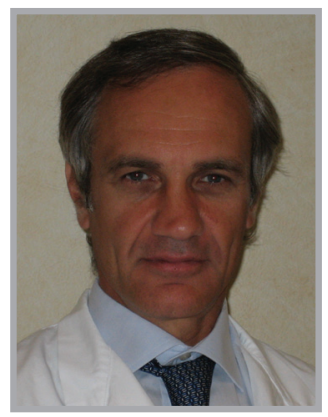

Francesco Lo-Coco* speaks to Sebastian Dennis-Beron, Commissioning Editor: Francesco Lo-Coco is currently a full professor of hematology and head of the Laboratory of Integrated Diagnosis of Oncohematologic Diseases at the Department of Biomedicine and Prevention of the University Tor Vergata of Roma. He obtained his MD degree from the University of Pisa in 1981, and his specialization in clinical and laboratory hematology is from the University La Sapienza of Rome in 1985. From 1992 to 1994, he has trained on molecular genetics of lymphomas at Columbia University, New York. His main scientific interest and research activities include genetic characterization, monitoring and treatment of hematologic tumors, particularly acute myeloid leukemia and acute promyelocytic leukemia (APL). He has published over 390 internationally peer-reviewed articles, mainly focused on molecular diagnosis and follow-up of leukemia as well as on treatment of APL. He chairs at present the APL subcommittee of the Italian National Cooperative Group, GIMEMA. He served as president of the Italian Society of Experimental Hematology, chairman of the Education Committee of the European Hematology Association, board member of the Italian Foundation for Cancer Research, member of the Committe on Health Research of the Italian Ministry of Health and member of the Editorial Board of the journals Leukemia, Journal of Clinical Oncology and Haematologica.

Accepted for publication: 5 December 2016; Published online: 13 January 2017

\section{KEYWORDS}

- acute promyelocytic leukemia

- arsenic trioxide $\bullet$ retinoic acid

- Trisenox ${ }^{\circledast}$ 
Q How have you seen the management of acute promyelocytic leukemia develop over the last decade?

I would consider myself a very lucky person as I was able to witness almost the entire history of acute promyelocytic leukemia (APL) over the past three decades. When I started with professor F Mandelli, who had been my mentor in the early 1980s, most of our patients died early as a result of hemorrhage and only few patients were cured. Over the past three decades as I said, I have had the chance to go through all the progress that has been done, initially with retinoic acid in the late 1980s. In early 1990s, we began combining retinoic acid, a vitamin A derivative, with conventional anthracycline chemotherapy and there was a dramatic improvement in patient outcomes, with over $70 \%$ long-term survival. But the real amazing progress of the last decade has been due to the advent of arsenic trioxide, which when given in association with all-trans retinoic acid (ATRA), has the possibility of actually curing most APL patients without any chemotherapy. As I said, I am a lucky investigator because I had the chance to see all of this treatment revolution develop.

Q How important or significant is the recent Committee for Medicinal Products for Human Use recommendation of an indication extension for the use \& availability of Trisenox ${ }^{\circledast}$ ?

I think this is a historic decision. It is extremely important for a number of reasons: first of all, this is the first time that we are able to cure an acute leukemia subset without the use of any chemotherapy; and the approval of a regimen that is based on two agents, retinoic acid and arsenic trioxide, represents a new paradigm that should be considered an example of the possibility to eradicate leukemia stem cells and to cure a hematologic tumor without the use of any chemotherapeutics at all. Another important reason I should mention is the fact that the favorable opinion that led to this extraordinary approval, is not based on an industry-driven protocol or a sponsored protocol, but rather on academic studies.

\section{Q What does this mean for patients in Europe?}

This means that patients with this disease and more specifically patients with low-to-standard risk APL - a group that makes up approximately $70-75 \%$ of patients with this disease - can have access to an important drug, which has been demonstrated to be superior to conventional chemotherapy and is thus an important treatment strategy. In practical terms, these patients all over Europe will receive a treatment that not only has greater efficacy than chemotherapy but is also associated with much less toxicity. In other words, we can avoid some serious risks in these patients such as infections, death in remission and the development of secondary leukemia, all of which are associated with chemotherapy.

Q Can you tell us a little bit more about the APL0406 study?

APL0406 (NCT00482833) is a randomized study that was started in 2007 by the Italian National Cooperative Group, GIMEMA, and was joined soon after by two German Cooperative Groups: the German-Austrian Acute Myeloid Leukemia Study Group and the Study Alliance Leukemia. In those years, toward the mid-2000s, there were basically two scenarios for treating of APL; we initially had very good results with the strategy of combining ATRA and chemotherapy. This strategy provided some $70-75 \%$ cure rates in the long term. However, it was and is still associated with considerable toxicity, such as infection, secondary leukemia and severe myelosuppression. This notwithstanding, we had very solid data for this strategy in the long term with huge patient numbers in these studies being treated and observed for prolonged follow-up periods.

At this time another scenario was emerging, where arsenic trioxide was being demonstrated as a very effective drug against APL, initially from China, followed by studies from India and then from the USA. In the USA, there was an idea brought up by E Estey from The University of Texas MD Anderson Cancer Center (TX, USA), of using arsenic trioxide in combination with ATRA as an alternative strategy to chemotherapy. There were promising data for this more experimental strategy, however, there were very small patient numbers included in the preliminary study and the follow-up was not prolonged. We then developed the idea of comparing the two sets of therapies in a randomized fashion. In this Phase III international comparative study, we included patients with low- to intermediate-risk APL.

The study was selected for the plenary session of the 54th American Society of Hematology and I had the privilege of presenting this data in December of 2012 in Atlanta. Some months 
after, the results were published in the New England Journal of Medicine [1], showing a significantly better outcome for patients receiving the chemotherapy-free regimen with arsenic trioxide and retinoic acid.

These results were recently updated and even in the long term, there is an increased advantage in terms of efficacy and safety for patients receiving this chemotherapy-free strategy [2].

\section{Q How significant are the event-free survival} rates measured in the trial?

We selected event-free survival as the primary objective in this trial because we thought that this gave a better estimate for assessing the role of arsenic trioxide in this specific disease. The event-free survival was significantly superior for patients receiving the chemotherapy-free option compared with the ATRA and chemotherapy combination. When you measure event-free survival, you would not only include death from any cause but also relapses, and as a result we thought this was a better estimate. The reason for that being, that arsenic trioxide is currently used as a common rescue treatment for patients who relapse and is approved as a salvage therapy for relapsing APL. Therefore, we believed that this may actually confound the assessment of ATO efficacy if we use overall survival as a comparative estimate to assess this drug frontline, given that you may actually end up with compounding factors such as the use of arsenic trioxide in relapse, which is currently used as standard.

\section{Q Quality-of-life outcomes were measured} as secondary objectives: why were these measures selected \& how were they assessed?

This was one of the secondary objectives in our trial and I believe it was actually the primary objective in another study conducted in the UK [3]. This is an important outcome, particularly if you have a disease like APL where there are very few events and very few relapses even when, what is now considered the old strategy of chemotherapy and ATRA in combination, is used. We believed that perhaps having a patienthealth-assessed measurement of the quality of life (QoL) would have been important as you may actually ended up having an equal efficacy and safety profile for the therapy, but report a better QoL.

This QoL assessment was self-reported by the patients themselves; this was measured by EORTC-qlq-c30 questionnaires validated by the European Organisation for Research and Treatment of Cancer (EORTC). The forms, which were completed and returned by patients, were then analyzed with the results showing greater benefit for patients receiving the chemotherapy-free option. This QoL assessment is vital as it is now being considered by regulatory bodies more and more as a valid outcome that should be considered.

\section{Q Which patient profile would benefit the most from treatment with Trisenox?}

At this point in time, we have shown that patients with non-high-risk APL, so mainly patients with low- to intermediate-risk APL undergoing frontline treatment or newly diagnosed, could benefit the most from this chemo-free option of arsenic trioxide and ATRA. In the future, we will assess the value of this strategy for high-risk patients as well; nevertheless, we are still using arsenic trioxide in the relapse setting as it is currently approved for patients who relapse after chemotherapy.

\section{Q Do you have any personal experience of} using Trisenox in the clinic?

We have treated a huge number of patients with this combination; we started by treating relapsing APL patients following chemotherapy. More than a decade ago, we moved this to a frontline therapy and we were interested in exploring other patient settings, though we certainly need to carry out more investigations.

\section{Q What do you see as the next steps for the} development of Trisenox?

I think there are some patient settings we still need to consider. We will start a new randomized comparative study for high-risk APL where we will explore the role of arsenic trioxide as a frontline treatment in this patient group. These patients are at higher risk of both early death and relapse. We definitely believe that there is room for investigation in this particular disease category. We are also interested in exploring the role of arsenic in the elderly as well as in children. I think there are very few data on children for arsenic trioxide and this is an area in real need of clinical investigation.

\section{Q Could this extension lead to a new} chemotherapy-free treatment option for APL? Definitely, this is what we hoped for and we are very much eager to see its approval. This would lead to, as I mentioned before, a new paradigm; the cure of an acute leukemia without 
any chemotherapy at all. I think this is quite important to foster more studies on targeted treatments in human oncology.

Q Where do you see the field of APL research going in the future?

We still have some hurdles to face because there are still many patients who die early from hemorrhagic events, even before diagnosis is confirmed or in the very initial days after diagnosis. We need to recognize what is happening in the 'real world', because these results we have been discussing today are mainly from to patients entered in clinical trials. However, if you look at registry based or population studies that are published in Sweden or Canada, very well-developed countries, you realize there is still nowadays up to $30 \%$ early death in this disease. This may be perhaps due to late diagnoses or delayed treatment initiation or referral of patients to unexperienced institutions, or finally other issues and we are very much willing to analyze the problem in more detail. The other area that fascinates me is the possibility to actually study new combinatorial treatments that include arsenic trioxide, which could be potentially effective in other leukemic forms beyond APL.

I feel such great emotion over these days and weeks to see what has been achieved thanks to these studies in APL. To witness such a dramatic improvement in patients and also having as we have said, this paradigm, gives more hope not only to investigators but also to all European citizens about the possibility of curing more diseases through a targeted, less toxic and individualized approach.

\section{Update}

Following F Lo-Coco's interview, Teva announced it had obtained approval from the
European Commission to use Trisenox as a first-line treatment for low- to intermediate-risk APL. The decision followed the recommendation from Committee for Medicinal Products for Human Use from the EMA. The decision, based solely on academic data endorsing the benefit for Trisenox, marks the treatment as the first chemo-free therapy for APL, which has huge significance for patients across Europe.

F Lo-Coco commented "This approval by the European Commission is good news for APL patients as we now have access to a cure for an acute leukemia without using chemotherapy. Moreover, this decision is a very positive endorsement by the European Commission, as it was made based solely on published academic research and studies. From now on, patients with non-high-risk disease will have access to this chemotherapy-free regimen of Trisenox plus retinoic acid combination as a frontline therapy. This has the potential to increase survival rates while minimizing side effects associated with chemotherapy."

\section{Disclaimer}

The opinions expressed in this interview are those of the interviewee and do not necessarily reflect the views of Future Medicine Ltd.

Financial \& competing interests disclosure $F$ Lo-Coco declares the following financial relationships: LUNDBECK - Honoraria, Advisory Board membership, Speaker's Bureau; TEVA: Honoraria, Advisory Board membership, Speaker's Bureau. The author has no other relevant affiliations or financial involvement with any organization or entity with a financial interest in or financial conflict with the subject matter or materials discussed in the manuscript apart from those disclosed.

No writing assistance was utilized in the production of this manuscript.

\section{References}

1 Lo-Coco F, Avvisati G, Vignetti M et al. Retinoic acid and arsenic trioxide for acute promyelocytic leukemia. N. Engl. J. Med. 369(2), 111-121 (2013).

2 Platzbecker U, Avvisati G, Cicconi L et al. Improved outcomes with retinoic acid and arsenic trioxide compared with retinoic acid and chemotherapy in non-high-risk acute promyelocytic leukemia: final results of the randomized Italian-German APL0406 trial. J. Clin. Oncol. doi:10.1200/JCO.2016.67.1982 (Epub ahead of print) (2016).
3

Burnett AK, Russell NH, Hills RK et al. Arsenic trioxide and all-trans retinoic acid treatment for acute promyelocytic leukaemia in all risk groups (AML17): results of a randomised, controlled, Phase III trial. Lancet Oncol. 16(13), 1295-1305 (2015). 